\title{
CARATHÉODORY-FEJÉR INTERPOLATION ON POLYDISKS
}

\author{
Jörg Eschmeier, Linda Patton and Mihai Putinar
}

\section{Introduction}

The question of whether a polynomial can be extended with higher degree terms to a convergent series from the unit disk $\mathbf{D}$ into itself is known as the Carathéodory-Fejér problem. The solvability of this problem is equivalent to the positive semi-definiteness of an associated Toeplitz type matrix which depends only on the coefficients of the original polynomial. Due to a fundamental observation of Sarason [12], the Carathéodory-Fejér problem, and its relative, the Nevanlinna-Pick problem, are now accessible via Hilbert space methods; more precisely these problems were reduced to a dilation scheme of linear operators. The commutant lifting theorem of Sz. Nagy and Foiaş is the abstract version of this dilation scheme; its numerous applications to function theory and engineering are well illustrated by the monograph [8].

Comparatively little is known in several complex variables. The integral representation formula for bounded analytic functions in classical domains of $\mathbf{C}^{n}$, known after its classical one variable version as the Herglotz-Nevanlinna formula, is now well understood, cf. [3], [10] and [13]. Via this formula, a characterization in positivity terms of the coefficients of the power series of an analytic function from $\mathbf{D}^{n}$ to $\mathbf{D}$ was derived by Korányi and Pukánszky [10], see also [3] and [13]. Other partial results towards a solution of the Carathéodory-Fejér problem in the polydisk are contained in Pfister's thesis [11] and, for low degree or special polynomials, in the works of the Russian school, see [3], [6] and the references contained there.

In what follows we discuss separately the case $n=2$, which is special due to the dilation theorem for commuting contractions of Ando; see [8]. Agler recently developed a successful Hilbert space theory which complements the function theory in the bidisk, with the special aim at solving the NevanlinnaPick interpolation problem; see [1], [2], and the references therein. A different approach to the same problem is due to Cole and Wermer [5].

In the present note we propose a solution to the Carathéodory-Fejér problem in the bidisk via Agler's version of the Herglotz-Nevanlinna formula. In vague terms, our main result asserts that the necessary and sufficient condition for solving this problem in degree $d$ is to truncate the Agler-Herglotz-Nevanlinna

Received July 1, 1998.

1991 Mathematics Subject Classification. 32A07, 41A05, 47A20.

Partially supported by the National Science Foundation Grant DMS-9800666. 
formula modulo terms of degree $d+1$ or higher. We parametrize all solutions of the Carathéodory-Fejér problem in terms of all unitary extensions of an isometry between finite dimensional spaces (a recurrent theme in the theory of commutant liftings). We prove that a rational function always exists among the extensions. As in all other similar results in dimension two or higher, the statements and their proofs contain a non-constructive part which makes all applications considerably more difficult than in the case of a single complex variable.

In higher dimensions Ando's theorem fails and we can rely only on the Herglotz-Nevanlinna formula. By slightly improving the main result of [10], we prove below that if the interpolation data are given on a large enough set, then the necessary positivity condition deduced from the Herglotz-Nevanlinna formula is also sufficient.

Section 2 below contains the main results and a few of their ramifications. Section 3 is devoted to proofs.

Acknowledgements. We thank J. W. Helton for his comments on potential applications of such interpolation results, cf. [9]. We are indebted to D. Timotin for timely informing us, while our note was circulating as a preprint, about his joint work [4] on similar questions.

\section{Main Results}

Let $n>1$ be a fixed integer, and let $\mathbf{T}^{n}$ denote the $n$-dimensional torus, regarded as the distinguished boundary of the polydisk $\mathbf{D}^{n}$. Let $f$ be an analytic function on $\mathbf{D}^{n}$. We denote by $H$ a separable, complex Hilbert space. We will encounter two conditions on the real part of $f$ :

$(\mathrm{HN})$ There exists a vector valued analytic function $g: \mathbf{D}^{n} \longrightarrow H$ such that

$$
f(z)+\overline{f(z)}=\prod_{j=1}^{n}\left(1-\left|z_{j}\right|^{2}\right)\|g(z)\|^{2}, \quad z \in \mathbf{D}^{n},
$$

and,

(AHN) There are vector valued analytic functions $g_{j}: \mathbf{D}^{n} \longrightarrow H, 1 \leq j \leq n$, such that

$$
f(z)+\overline{f(z)}=\sum_{j=1}^{n}\left(1-\left|z_{j}\right|^{2}\right)\left\|g_{j}(z)\right\|^{2}, \quad z \in \mathbf{D}^{n} .
$$

Condition (HN) is equivalent to the fact that $\operatorname{Re} f(z) \geq 0$ for $z \in \mathbf{D}^{n}$; in this case the non-tangential boundary values of $\operatorname{Re} f(z)$ exist at points of $\mathbf{T}^{n}$ and give there a positive measure $\mu$ with non-zero Fourier coefficients only in the positive octant and its opposite. The converse also holds and

$$
\|g(z)\|^{2}=\int_{\mathbf{T}^{n}} \frac{d \mu(\zeta)}{\prod_{j=1}^{n}\left|1-\zeta_{j} \overline{z_{j}}\right|^{2}}
$$

cf. [10] and [13].

On the other hand, condition (AHN) is equivalent to the fact that $\operatorname{Re} f(T) \geq 0$ for every commutative $n$-tuple of strictly contractive operators. Due to Ando's 
theorem, for $n=2$ the two conditions are equivalent, see [1]. By replacing $f(z)+\overline{f(z)}$ in the two conditions by $1-|f(z)|^{2}$ one obtains the corresponding representations of analytic maps from $\mathbf{D}^{n}$ into $\mathbf{D}$.

For a positive integer $d$ and two polynomials $P(z, \bar{z}), Q(z, \bar{z})$ we denote in the sequel $P \equiv Q \bmod \left(z^{d+1}, \bar{z}^{d+1}\right)$ if the difference $P(z, \bar{z})-Q(z, \bar{z})$ contains only monomials of degree $d+1$ or higher in at least one set of variables $z$ or $\bar{z}$. The same definition can be applied to holomorphic functions in the bidisk by using power series expansions about zero.

Theorem 1. Let $d$ be a positive integer and let $P(z)$ be a polynomial of degree less than or equal to $d$ in two complex variables. There exists an analytic function $F: \mathbf{D}^{2} \longrightarrow \mathbf{D}$ such that $P \equiv F \bmod \left(z^{d+1}\right)$ if and only if there are Hilbert spaces $H_{1}$ and $H_{2}$ and a pair of vector valued polynomial functions of degree less than or equal to $d, A_{k}: \mathbf{D}^{2} \longrightarrow H_{k}, k=1,2$, such that:

$$
\begin{aligned}
1-P(z) \overline{P(z)} & \equiv \\
& \left(1-\left|z_{1}\right|^{2}\right)\left\|A_{1}(z)\right\|_{1}^{2}+\left(1-\left|z_{2}\right|^{2}\right)\left\|A_{2}(z)\right\|_{2}^{2} \bmod \left(z^{d+1}, \bar{z}^{d+1}\right) .
\end{aligned}
$$

The Hilbert spaces $H_{1}$ and $H_{2}$ in the statement may not be identical and can each be chosen of finite dimension less than or equal to $(d+1)(d+2) / 2$. Note that \|\|$_{k}$ and $\langle,\rangle_{k}$ denote the norm and inner product, respectively, on $H_{k}$ for $k=1,2$.

The proof of Theorem 1, which will be given in the next section, provides also a matricial parametrization of all extensions $F$. Such a parametrization has already been discussed in [2] for the Nevanlinna-Pick problem on the bidisk. A direct consequence of the proof of Theorem 1 is the next corollary.

Corollary 1. In the conditions of Theorem 1, there always exists an extension $F$ which is a rational function of degree less than or equal to $d^{2}+3 d+2$.

As a matter of fact, Theorem 1 holds in any dimension $n \geq 2$, but only for analytic functions $F$ in the polydisk which satisfy $\|F(T)\| \leq 1$ for all commutative $n$-tuples of strict contractions $T$, cf. [1].

Let $\left(d_{1}, d_{2}\right)$ be a pair of positive integers, and assume that the given polynomial $P(z)$ has degree $d_{1}$ in $z_{1}$ and $d_{2}$ in $z_{2}$, respectively. Then we say that the bidegree of $P$ is less than or equal to $\left(d_{1}, d_{2}\right)$. Pfister's method [11] gives necessary and sufficient conditions for the existence of a rational extension $F$, modulo a fixed bidegree, of the form $F=Q^{\sharp} / Q$, where $Q$ is a polynomial of bidegree less than or equal to $\left(d_{1}, d_{2}\right)$ which has no zeroes in the bidisk, and

$$
Q^{\sharp}(z)=z_{1}{ }^{d_{1}} z_{2}{ }^{d_{2}} \overline{Q\left(\frac{1}{\bar{z}}\right)} .
$$

See also [6]. Unfortunately, as Pfister points out, such a special rational solution does not always exist.

For the next interpolation result in higher dimensions we need to define a uniqueness point for a subset of $\mathbf{D}^{n}$ where $n$ is an arbitrary positive integer. If 
$p \in \mathbf{C}^{n}$ then let $B(p, r)$ denote the ball with center $p$ and radius $r$. Let $K$ be a subset of $\mathbf{D}^{n}$ and let $p \in K$. We say $p$ is a uniqueness point of $K$ if the following implication holds for all $r \in(0,1)$ : If $g$ and $h$ are analytic functions on $B(p, r)$ and

$$
h(z)=g(z) \quad \text { for all } z \in B(p, r) \cap K,
$$

then

$$
h(z)=g(z) \quad \text { for all } z \in B(p, r) .
$$

Recall that a function $h$ defined on $\mathbf{D}^{n} \times \mathbf{D}^{n}$ is non-negative definite if for any positive integer $m$ and points $z_{1}, \ldots, z_{m}$ in $\mathbf{D}^{n}$, the expression

$$
\sum_{j=1}^{m} \sum_{i=1}^{m} h\left(z_{i}, z_{j}\right) v_{i} \bar{v}_{j}
$$

is non-negative for all vectors $v \in \mathbf{C}^{m}$.

Theorem 2. Let $K$ be a subset of $\mathbf{D}^{n}$ which contains at least one uniqueness point. If $f$ is a function on $K$ with the property that the kernel

$$
\frac{1-f(z) \overline{f(w)}}{\prod_{k=1}^{n}\left(1-z_{k} \overline{w_{k}}\right)}, \quad z, w \in K,
$$

is non-negative definite, then there exists an analytic function $F: \mathbf{D}^{n} \longrightarrow \mathbf{D}$ which coincides with $f$ on $K$.

This is a refinement of [10], where the set $K$ has the following property: there exists a point $\omega \in \mathbf{D}^{n}$ such that $z \in K$ implies that the point $\tilde{z}$ is also in $K$, where $\tilde{z}$ is obtained by replacing any coordinate of $z$ with the corresponding coordinate of $\omega$. Otherwise, we shall see that the proof is very similar. Note that there are simple examples of subsets of $\mathbf{D}^{n}$ from which bounded analytic functions do not have analytic extensions to $\mathbf{D}^{n}$ which are still bounded. To be more specific, such an example is the analytic set $\left\{(z, w) ; w^{2}=z(z-1)^{2}\right\}$ in the unit polydisk in the two variables $z$ and $w$; see [7] for details.

\section{Proofs}

Proof of Theorem 1. The necessity part of the proof follows from the (AHN) formula above, by taking its reduction modulo $|z|^{d+1}$.

For the sufficiency part of the proof, we need the following terminology: let $a=\left\{a_{\alpha}\right\}_{|\alpha|=0}^{d}$ be a bi-indexed finite sequence of vectors in a Hilbert space $H$ with $\alpha=\left(\alpha_{1}, \alpha_{2}\right)$ and $|\alpha|=\alpha_{1}+\alpha_{2}$. Define the shift operators $S_{1}^{*}$ and $S_{2}^{*}$ on the sequence $a$ by

$$
\left(S_{1}^{*} a\right)_{\alpha}= \begin{cases}a_{\alpha-(1,0)} & \text { if } \alpha_{1}>0 \\ 0 & \text { if } \alpha_{1}=0\end{cases}
$$

and

$$
\left(S_{2}^{*} a\right)_{\alpha}= \begin{cases}a_{\alpha-(0,1)} & \text { if } \alpha_{2}>0 \\ 0 & \text { if } \alpha_{2}=0\end{cases}
$$


for all $\alpha$ with $|\alpha| \leq d$.

Now let $P(z)=\sum_{|\alpha|=0}^{d} c_{\alpha} z^{\alpha}$ and assume there exist $A_{1}$ and $A_{2}$ satisfying (1) with expansions

$$
A_{k}(z)=\sum_{|\alpha|=0}^{d} a_{\alpha}^{(k)} z^{\alpha} \quad \text { for } k=1,2 .
$$

We will be applying $S_{k}^{*}$ to the finite sequence $a^{(k)} \equiv\left\{a_{\alpha}^{(k)}\right\}_{|\alpha|=0}^{d}$ of coefficients of $A_{k}$ for $k=1,2$. Note that $S_{k}^{*} a^{(k)}$ is a bi-indexed finite sequence of vectors in $H_{k}$, while for each bi-index $\alpha,\left(S_{k}^{*} a^{(k)}\right)_{\alpha} \in H_{k}$.

By expanding (1) and equating coefficients of like terms, we obtain

$$
1-\left|c_{00}\right|^{2}=\left\|a_{00}^{(1)}\right\|_{1}^{2}+\left\|a_{00}^{(2)}\right\|_{2}^{2}
$$

and

$$
-c_{\alpha} \overline{c_{\beta}}=\left(\sum_{k=1}^{2}\left\langle a_{\alpha}^{(k)}, a_{\beta}^{(k)}\right\rangle_{k}\right)-\left(\sum_{k=1}^{2}\left\langle\left(S_{k}^{*} a^{(k)}\right)_{\alpha},\left(S_{k}^{*} a^{(k)}\right)_{\beta}\right\rangle_{k}\right),
$$

where (3) holds for all nonzero $(\alpha, \beta)$ where $|\alpha|$ and $|\beta|$ are each less than or equal to $d$.

Equations (2) and (3) show that the map $V$ defined on a subspace of $\mathbf{C} \oplus$ $H_{1} \oplus H_{2}$ by

$$
V(1 \oplus 0 \oplus 0)=c_{00} \oplus a_{00}^{(1)} \oplus a_{00}^{(2)},
$$

and

$$
V\left(0 \oplus\left(S_{1}^{*} a^{(1)}\right)_{\alpha} \oplus\left(S_{2}^{*} a^{(2)}\right)_{\alpha}\right)=c_{\alpha} \oplus a_{\alpha}^{(1)} \oplus a_{\alpha}^{(2)}, \quad 0<|\alpha| \leq d,
$$

is an isometry.

Pick a unitary extension $U$ of $V$, still acting on $\mathbf{C} \oplus\left[H_{1} \oplus H_{2}\right]$, and let us denote:

$$
U=\left(\begin{array}{ll}
U_{11} & U_{12} \\
U_{21} & U_{22}
\end{array}\right)
$$

with respect to the decomposition into $\mathbf{C}$ and $\left[H_{1} \oplus H_{2}\right]$, respectively.

Equations (4) and (5) can be expressed in terms of $U$ as follows:

$$
\left\{\begin{array}{cl}
U_{11} & =c_{00}, \\
U_{21} & =a_{00}^{(1)} \oplus a_{00}^{(2)}, \\
U_{12}\left(\left(S_{1}^{*} a^{(1)}\right)_{\alpha} \oplus\left(S_{2}^{*} a^{(2)}\right)_{\alpha}\right) & =c_{\alpha}, \quad 0<|\alpha| \leq d, \\
U_{22}\left(\left(S_{1}^{*} a^{(1)}\right)_{\alpha} \oplus\left(S_{2}^{*} a^{(2)}\right)_{\alpha}\right) & =a_{\alpha}^{(1)} \oplus a_{\alpha}^{(2)}, \quad 0<|\alpha| \leq d .
\end{array}\right.
$$

Let $P_{k}$ be the orthogonal projection of $H_{1} \oplus H_{2}$ onto $H_{k}$ and introduce the operator-valued map:

$$
D(z)=z_{1} P_{1} \oplus z_{2} P_{2}, \quad\left(z_{1}, z_{2}\right) \in \mathbf{D}^{2} .
$$

Now we can define the function $F$ on the bidisk which will satisfy the Carathéodory-Fejér conditions. Let

$$
F(z)=U_{11}+U_{12} D(z)\left(I-U_{22} D(z)\right)^{-1} U_{21} .
$$


Obviously, $F$ is analytic in the bidisk; less obvious, but elementary, is the observation that $|F(z)| \leq 1$ for $z$ in the bidisk. For a proof see [2] or let $v(z)=$ $\left(I-U_{22} D(z)\right)^{-1} U_{21}$ and consider the linear system:

$$
U\left(\begin{array}{c}
1 \\
D(z) v(z)
\end{array}\right)=\left(\begin{array}{c}
F(z) \\
v(z)
\end{array}\right)
$$

This means (since $U$ is unitary)

$$
|F(z)|^{2}+\|v(z)\|_{H_{1} \oplus H_{2}}^{2}=1+\|D(z) v(z)\|_{H_{1} \oplus H_{2}}^{2} .
$$

Multiplication by $D(z)$ in $H_{1} \oplus H_{2}$ is a contraction for each $z \in \mathbf{D}^{2}$. Thus

$$
|F(z)|^{2}+\|v(z)\|_{H_{1} \oplus H_{2}}^{2} \leq 1+\|v(z)\|_{H_{1} \oplus H_{2}}^{2}
$$

which implies that $|F(z)| \leq 1$ for $z \in \mathbf{D}^{2}$.

It remains to show that $F \equiv P \bmod \left(z^{d+1}\right)$. Note that equality $\bmod \left(z^{d+1}\right)$ of polynomials with coefficients in $H_{1} \oplus H_{2}$ is preserved under multiplication by $D(z)$ or any operator independent of $z$. A calculation using this fact and (6) shows that

$$
\left(I-U_{22} D(z)\right)\left(A_{1}(z) \oplus A_{2}(z)\right) \equiv a_{00}^{(1)} \oplus a_{00}^{(2)} \quad \bmod \left(z^{d+1}\right),
$$

which implies that

$$
\left(I-U_{22} D(z)\right)^{-1}\left(a_{00}^{(1)} \oplus a_{00}^{(2)}\right) \equiv A_{1}(z) \oplus A_{2}(z) \bmod \left(z^{d+1}\right) .
$$

Thus

$$
\begin{aligned}
F(z) & \equiv c_{00}+U_{12} D(z)\left(A_{1}(z) \oplus A_{2}(z)\right) \\
& \equiv c_{00}+U_{12}\left(\left(\sum_{|\alpha|=1}^{d}\left(S_{1}^{*} a^{(1)}\right)_{\alpha} z^{\alpha}\right) \oplus\left(\sum_{|\alpha|=1}^{d}\left(S_{2}^{*} a^{(2)}\right)_{\alpha} z^{\alpha}\right)\right) \\
& \equiv c_{00}+\sum_{|\alpha|=1}^{d} c_{\alpha} z^{\alpha} \quad \bmod \left(z^{d+1}\right) .
\end{aligned}
$$

The last equivalence follows from (6) and concludes the proof of the theorem.

For the proof of Corollary 1, it remains to remark that we can always choose the Hilbert spaces $H_{1}$ and $H_{2}$ of finite dimension $D$, less than or equal to the number of coefficients $c_{\alpha}$, where $|\alpha| \leq d$. That is, $D \leq(d+1)(d+2) / 2$. Thus, the operator $D(z)\left(I-U_{22} D(z)\right)^{-1}$ in $(7)$ can be expressed as a $\left(d^{2}+3 d+2\right) \times$ $\left(d^{2}+3 d+2\right)$ matrix with entries which are rational functions of $z$. These rational functions have identical denominators and each rational function has degree at most $d^{2}+3 d+2$. Therefore, the function $F$ will be rational of degree at most this integer.

The above proof gives a parametrization of all solutions $F$ to the Carathéodory-Fejér problem. Namely, pick a decomposition (1), with auxiliary Hilbert spaces $H_{1}, H_{2}$; then define by formulas (4) and (5) the isometric map $V$ on a subspace of $\mathbf{C} \oplus\left[H_{1} \oplus H_{2}\right]$ and then choose an extension of $V$ to a unitary map 
$U$, on the same space. As a result, it turns out that, for the fixed decomposition (1), formula (7) gives all possible solutions $F$. If it happens that the map $V$ is already defined on the whole space, then the solution to the Carathéodory-Fejér problem is unique within the specific minimal choice of $H_{1}, H_{2}$. Compare with the one-variable case [8].

These techniques could be used to combine the Nevanlinna-Pick and Carathéodory-Fejér problems on the bidisk by finding conditions that are equivalent to the existence of a bounded analytic function whose Taylor expansion is specified to a certain degree at several points in the bidisk. For more details about such results, and their relation with the commutant lifting theorem, see [4].

Proof of Theorem 2. The proof is similar, at least in principle, to the proof of the main result in [10]. We merely indicate the points of the proof which are different from those in [10]. By standard Möbius transforms we can assume that $p=0$ is the uniqueness point of $K$ and that $f$ maps $K$ onto the right half plane $\mathbf{C}_{+}$rather than the unit disk.

We introduce some notation involving the set $\mathbf{N}^{n}$ of multi-indices of length $n$. Define

$$
O(n)=\left\{\alpha \in \mathbf{N}^{n}|| \alpha \mid \leq n, \alpha_{i}=0 \text { or } \alpha_{i}=1 \quad \forall 1 \leq i \leq n, \text { and }|\alpha| \text { is odd }\right\} .
$$

$E(n)$ is defined similarly but only contains $\alpha$ such that $|\alpha|$ is even and strictly positive. Let $N$ denote the cardinality of $O(n)$.

Let $H$ be a complex separable Hilbert space which carries the factorization of the non-negative kernel in the statement of the theorem :

$$
\frac{f(z)+\overline{f(w)}}{\prod_{k=1}^{n}\left(1-z_{k} \overline{w_{k}}\right)}=\langle e(z), e(w)\rangle, \quad z, w \in K
$$

The function $e$ maps $K$ into $H$ and we can assume that $H$ is spanned by the values of $e$.

Alternately setting $z$ and $w$ equal to 0 in equation (8) above results in the identity:

$$
\prod_{k=1}^{n}\left(1-z_{k} \overline{w_{k}}\right)\langle e(z), e(w)\rangle=\langle e(z), e(0)\rangle+\langle e(0), e(w)\rangle-\langle e(0), e(0)\rangle,
$$

which holds for all $z$ and $w$ in $K$ and which in turn (after expanding the product) implies

$$
\begin{aligned}
\sum_{\alpha \in O(n)}\left\langle z^{\alpha} e(z), w^{\alpha} e(w)\right\rangle & = \\
& \left(\sum_{\alpha \in E(n)}\left\langle z^{\alpha} e(z), w^{\alpha} e(w)\right\rangle\right)+\langle e(z)-e(0), e(w)-e(0)\rangle,
\end{aligned}
$$

for all $z$ and $w$ in $K$.

We will use equation (10) to define an isometry which will be used to create an analytic function that extends $e$ to $\mathbf{D}^{n}$. 
Let $H^{N}$ denote the direct sum of $N$ copies of $H$. Let $S$ be the subset of $H^{N}$ consisting of all elements of the form $\bigoplus_{\alpha \in O(n)} z^{\alpha} e(z)$ for $z \in K$. Finally let $H_{o}^{N}$ be the vector subspace of $H^{N}$ consisting of all finite linear combinations of elements of $S$.

Define an operator $\tilde{V}$ from $H_{o}^{N}$ into $H^{N}$ by specifying $\tilde{V}$ on $S$ as

$$
\tilde{V}\left(\oplus_{\alpha \in O(n)} z^{\alpha} e(z)\right)=\left(\oplus_{\alpha \in E(n)} z^{\alpha} e(z)\right) \oplus(e(z)-e(0)) .
$$

and extending $\tilde{V}$ linearly to elements of $H_{o}^{N}$.

Note that the right side of equation (11) is in $H^{N}$ because the cardinality of $E(n)$ is $N-1$; therefore equation (10) shows that $\tilde{V}$ is an isometry from $H_{o}^{N}$ to a subspace of $H^{N}$. We can thus extend $\tilde{V}$ to a contraction $V$ on $H^{N}$.

Let $\alpha_{1}, \ldots, \alpha_{N}$ be a list of the elements of $O(n)$. Let $V=\left[V_{i, j}\right]_{i, j=1}^{N}$ be the block decomposition of $V$ such that each $V_{i, j}$ is a contraction on $H$.

When equation (11) is stated for the last row of the block decomposition, we obtain

$$
\sum_{i=1}^{N} V_{N, i} z^{\alpha_{i}} e(z)=e(z)-e(0), \quad z \in K
$$

Thus

$$
\left(I-\sum_{i=1}^{N} z^{\alpha_{i}} V_{N, i}\right) e(z)=e(0), \quad z \in K
$$

Since each $V_{N, i}$ is a contraction, there exists $r \in(0,1)$ such that the operator $\sum_{i=1}^{N} z^{\alpha_{i}} V_{N, i}$ is a contraction for all $z \in B(0, r)$. Therefore

$$
e(z)=\left(I-\sum_{i=1}^{N} z^{\alpha_{i}} V_{N, i}\right)^{-1} e(0)
$$

for all $z \in K \cap B(0, r)$, and the right side of equation (13) defines a function $E$ which is analytic for all $z$ in $B(0, r)$. If $\sum_{\alpha \in \mathbf{N}^{n}} c_{\alpha} z^{\alpha}$ is the Taylor expansion of $E$ at 0 , then this series converges to $E$ in $B(0, r)$.

Since $e(z)=E(z)$ for $z \in K \cap B(0, r)$, equation (9) shows that

$$
\prod_{k=1}^{n}\left(1-z_{k} \overline{w_{k}}\right)\langle E(z), E(w)\rangle=\langle E(z), e(0)\rangle+\langle e(0), E(w)\rangle-\langle e(0), e(0)\rangle
$$

for all $z \in K \cap B(0, r)$.

Fix $w \in K \cap B(0, r)$. If the uniqueness point property is applied to the analytic function of $z$ defining each side of equation (14), we obtain equation (14) for all $z \in B(0, r)$; likewise the identity holds for all $w \in B(0, r)$. We can thus substitute the Taylor series of $E$ into both sides of equation (14). Identifying the coefficients of $z^{\alpha} \bar{w}^{\alpha}$ that arise on each side of this equation yields

$$
\left\|c_{0}\right\|=\left\|c_{\alpha}\right\| \quad \text { for all } \alpha \in N^{n} \text {. }
$$


Therefore the multiradius of convergence for the Taylor series representation of the function $E$ is $(1, \ldots, 1)$, and analytic continuation in $z$ and $w$ yields equation (14) for all $z$ and $w$ in $\mathbf{D}^{n}$.

Now define

$$
F(z)=\langle E(z), e(0)\rangle-\overline{f(0)}, \quad z \in \mathbf{D}^{n} .
$$

$F$ is clearly a complex-valued analytic function on $\mathbf{D}^{n}$ since $E$ is a vectorvalued analytic function on $\mathbf{D}^{n}$. Furthermore, for $z \in K$ and $w=0$, equation (8) implies

$$
f(z)=\langle e(z), e(0)\rangle-\overline{f(0)}, \quad z \in K .
$$

Since $e(z)=E(z)$ for $z \in K$, it follows that $f(z)=F(z)$ for $z \in K$.

Finally,

$$
\begin{aligned}
\operatorname{ReF}(z) & =\langle E(z), e(0)\rangle+\langle e(0), E(z)\rangle-\overline{f(0)}-f(0) \\
& =\langle E(z), e(0)\rangle+\langle e(0), E(z)\rangle-\langle e(0), e(0)\rangle \\
& =\prod_{k=1}^{n}\left(1-\left|z_{k}\right|^{2}\right)\langle E(z), E(z)\rangle \geq 0 .
\end{aligned}
$$

The last equality follows from equation (14). This completes the proof of Theorem 2 .

A simple example contained in [10] shows that the uniqueness condition in Theorem 2 cannot be dropped from the hypothesis.

\section{References}

[1] J. Agler, On the representation of certain holomorphic functions defined on a polydisc, pp. 47-66, Oper. Theory Adv. Appl., 48, Birkhäuser Verlag, Basel, 1990.

[2] J. Agler and J. McCarthy, Nevanlinna-Pick interpolation on the bidisk, J. Reine Angew. Math 506 (1999), 191-204.

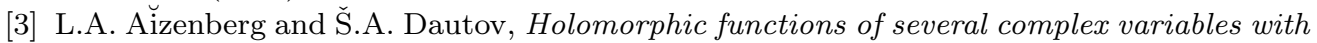
nonnegative real part, Math. USSR Sb. 28 (1976), 301-312.

[4] J. Ball, W. Li, D. Timotin, and T. Trent, A commutant lifting theorem on the polydisc, preprint 1998.

[5] B. Cole and J. Wermer, Pick interpolation, von Neumann inequalities, and hyperconvex sets, preprint 1996.

[6] Sh.A. Dautov and G. Khudaiberganov, On the Carathéodory-Fejér problem in multidimensional complex analysis, Sb. Mat. Zh. 23 (1982), 58-64.

[7] A. Douady, Le problème des modules pour les sous-espaces analytiques compacts d'un espace analytique donné, Ann. Inst. Fourier 16 (1966), 1-95.

[8] C. Foiaş and A.E. Frazho, The commutant lifting approach to interpolation problems, Operator Theory: Advances and Applications, 44, Birkhäuser Verlag, Basel, 1990.

[9] J.W. Helton, An application of several complex variables theory to linear model varying systems, preprint 1998.

[10] A. Korányi and L. Pukánszky, Holomorphic functions with positive real part on polycylinders, Trans. Amer. Math. Soc. 108 (1963), 449-456.

[11] A. Pfister, Über das Koeffizientenproblem der beschränkten Funktionen von zwei Veränderlichen, Math Ann. 146 (1962), 249-262.

[12] D. Sarason, Generalized interpolation in $H^{\infty}$, Trans. Amer. Math. Soc. 127 (1967), 179203. 
[13] V.S. Vladimirov, Holomorphic functions with positive imaginary part in the future tube $I V$, Mat. Sb. (N.S.) 104 (146) (1977), 341-370.

Fachbreich Mathematik, Universität des Saarlandes, Postfach 1511 50, D-66041, SAARBRÜCKEN, GERMANY

E-mail address: eschmei@math.uni-sb.de

Department of Mathematics, California Polytechnical State University, San Luis OBISPO, CA 93407

E-mail address: lpatton@calpoly.edu

Department of Mathematics, University of California, Santa Barbara, CA 93106

E-mail address: mputinar@math.ucsb.edu 